\title{
Semiclassical Mechanics of Rotons
}

\author{
T. M. Sanders, Jr. *
}

October 31, 2018

\begin{abstract}
The elementary excitations in superfluid liquid ${ }^{4} \mathrm{He}$ named rotons have an unusual dispersion curve. The energy is an approximately quadratic function of $\left(p-p_{0}\right)$, the difference between the magnitude of the momentum $p$ and a characteristic value $p_{0}$. As a result, while for $p>p_{0}$ a roton has its (group) velocity parallel to its momentum, when $p<p_{0}$ the velocity and momentum are antiparallel. When $p=p_{0}$, the roton has non-zero momentum but zero velocity. These kinematic properties lead to unusual trajectories when rotons scatter or experience external forces. This paper examines this behavior in the classical (ray optics) limit, where the roton wavelength is small compared with all other dimensions. Several experiments illustrate these effects. The examples are interesting in themselves, and also offer unconventional pedagogical possibilities.
\end{abstract}

\section{Introduction}

\section{$1.1 \quad$ Background}

The concept of elementary excitations in superfluid liquid helium $\left({ }^{4} \mathrm{He}\right)$ was introduced by L. D. Landau in 1941. [1] The essential idea of Landau is that the low-lying excited states of the macroscopic system have energies $E\left(E_{0}\right.$ is the ground state energy.) and momentum $\vec{P}$ which can be expressed in the form

*Author's address: H. M. Randall Laboratory, University of Michigan, Ann Arbor, MI 48109-1120, USA. 


$$
\begin{gathered}
E=E_{0}+\sum_{\vec{p}} n(\vec{p}) \varepsilon(\vec{p}) \\
\vec{P}=\sum_{\vec{p}} n(\vec{p}) \vec{p} .
\end{gathered}
$$

That is, the system of strongly-interacting particles (He atoms) is replaced by a collection of non (or weakly) interacting "elementary excitations" or "quasiparticles." In Landau's paper of 1941, he suggested a form for the dispersion curve (the relation between energy $\varepsilon$ and momentum $\vec{p}$ ) for these entities. For an isotropic system, such as a liquid, the energy can depend only on $p$, the magnitude of the momentum. The 1941 dispersion curve has two branches: There is an "acoustic" branch with $\varepsilon(p)=c p$, representing longitudinal phonons ( $c$ is the speed of sound.), and a second branch with $\varepsilon(p)=p^{2} / 2 \mu+\Delta . \quad$ Landau called these elementary excitations "rotons." The situation seems reminiscent of that in a solid, with its acoustical and optical branches. There is, however, an important difference: The liquid has complete translational invariance (not just against finite translations). Thus the momentum of the elementary excitations is not just a quantum number like crystal momentum, but is the usual physical momentum. Landau evaluated the two free parameters $\Delta$ and $\mu$ by fitting measured values of the specific heat of the liquid, and used the model to calculate the normal and superfluid fractions as functions of the temperature.

In his brief 1947 paper, [2] Landauf modified the relationship $\varepsilon(p)$ in order to account for some experimental data on the propagation of second sound. The curve he drew in 1947 agrees remarkably well with modern determinations of $\varepsilon(p)$, using inelastic neutron scattering. The two branches are replaced by a single branch which has a linear part, a maximum, and a minimum: We call the elementary excitations near the origin "phonons", those near the minimum "rotons." These two parts of the spectrum are dominant in determining the low-temperature properties of liquid helium. Donnelly [5] has written a recent introduction to the subject with interesting historical perspective and some discussion of the microscopic nature of the roton, a subject beyond the scope of the present article.

\footnotetext{
${ }^{1}$ Landau [1] writes that "This name was suggested by I. E. Tamm"

${ }^{2}$ Landau's papers of 1941 and 1947 are reprinted in several books, such as [3] and [4]
} 
Landau emphasized the importance of the finite slope of the dispersion curve at the origin - in distinction to the behavior of the Ideal Bose Gas. A consequence of this finite slope is a "critical velocity", below which the flow of the helium is dissipationless. In the simplest case the primary dissipation process would be roton emission. With rare exceptions [6] other dissipation mechanisms occur at velocities below that required for roton emission.

The reasons why there are no other states near the ground state of an interacting Bose system are considered in the papers of Feynman [7] in the 1950s.

\subsection{Current interest}

Since the development of the dilution refrigerator, it has become practical to perform experiments on "ballistic rotons." That is, at sufficiently low temperature the mean free path of a roton becomes as long as the size of the apparatus. Rotons (and phonons) are produced by a "heat pulse" in the liquid or, in some cases, by helium atoms incident on the surface of the liquid from the (low-density) vapor above it. We will consider such experiments in sections 6 and 8 .

\section{The dispersion curve}

The dispersion curve for elementary excitations in superfluid ${ }^{4} \mathrm{He}$, determined by inelastic neutron scattering [8]. is shown in figure 11. The region near the minimum is usually described by the equation

$$
\varepsilon(p)=\left(p-p_{0}\right)^{2} / 2 \mu+\Delta .
$$

At low temperature and pressure, approximate values for the parameters are: $q_{0}=p_{0} / \hbar=1.92 \AA^{-1}, \Delta / k_{B}=8.62 \mathrm{~K}, \mu=m_{4} / 6$. The function $\varepsilon(p)$ can be considered a Hamiltonian for the free roton, since $\partial \varepsilon / \partial p_{i}=v_{i}$ is a component of the (group) velocity of a roton wave packet. Using the expression (11), we have $\vec{v}=\hat{p}\left(p-p_{0}\right) / \mu$. 


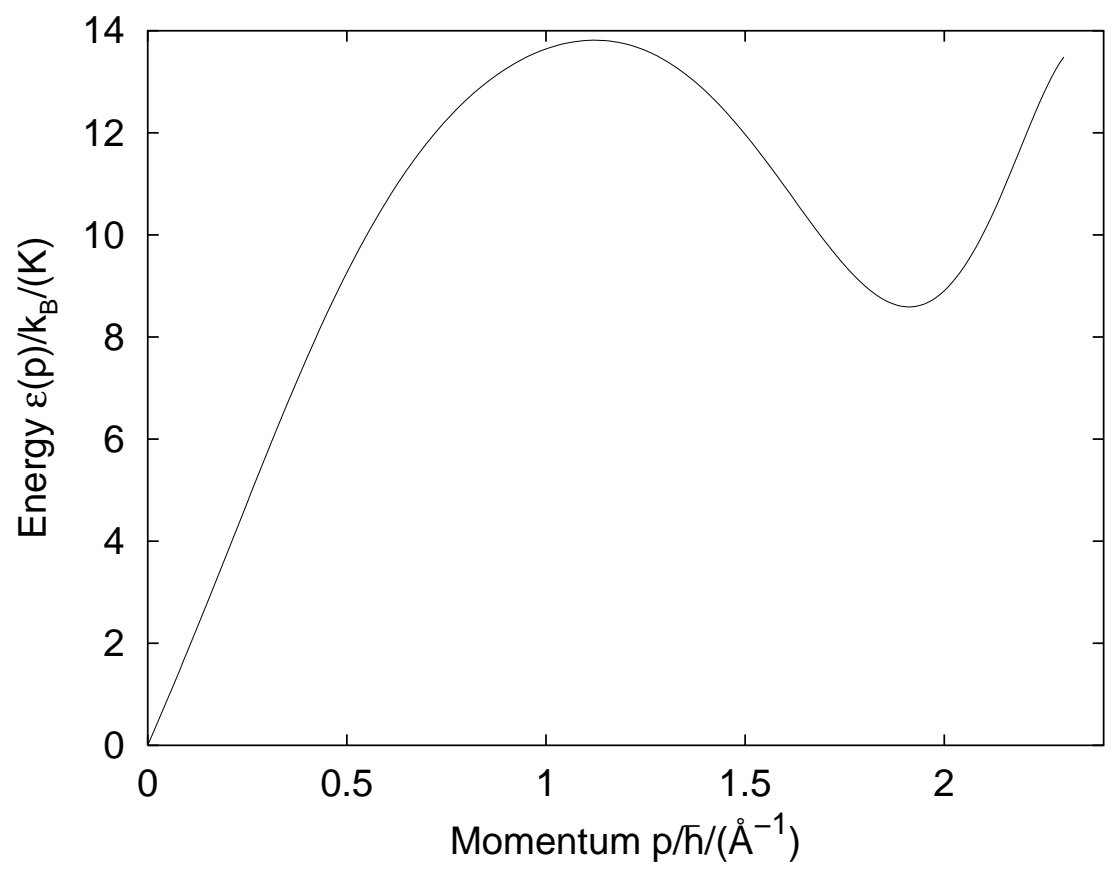

Figure 1: Dispersion curve for elementary excitations in liquid helium, calculated from the polynomial fit in reference [9]. The roton region is near the minimum. 


\subsection{Statistical Thermodynamics}

We recapitulate some results which we will later find useful. Since we will be interested in temperatures less than or of order $1 \mathrm{~K}$, we will always have $\varepsilon(p) \geq \Delta>>k_{B} T$, whence $\exp \left(-\varepsilon(p) / k_{B} T\right)=\exp (-\beta \varepsilon(p))<<1$ will always be valid. (The symbol $\beta$ stands, as is usual, for $1 / k_{B} T$.) This permits considerable simplification of various equations. Furthermore, all integrand functions of $p$ will have contributions peaked near $p=p_{0}$. Thus, we transform integrals over $p$ over the range from zero to infinity to integrals over $\left(p-p_{0}\right)$ from $-\infty$ to $\infty$.

The expected number of rotons in a given mode $\vec{p}$ :

$$
\langle n(\vec{p})\rangle=(\exp (\beta \varepsilon(p))-1)^{-1} \approx \exp (-\beta \varepsilon(p)) .
$$

The number density of rotons:

$$
n_{\rho} \equiv N_{\rho} / V=(2 \pi \hbar)^{-3} \int_{0}^{\infty} \exp (-\beta \varepsilon(p)) 4 \pi p^{2} d p
$$

The Helmholtz Free Energy:

$$
F_{\rho}=-k_{B} T V /(2 \pi \hbar)^{3} \int_{0}^{\infty} \exp (-\beta \varepsilon(p)) 4 \pi p^{2} d p
$$

The average "kinetic" energy per roton:

$$
\begin{aligned}
\left\langle\left(p-p_{0}\right)^{2} / 2 \mu\right\rangle & =\left\langle\mu v^{2} / 2\right\rangle \\
& =-\partial / \partial \beta\left(\log \int_{0}^{\infty} \exp \left(-\beta\left(p-p_{0}\right)^{2} / 2 \mu\right) p^{2} d p\right) \\
& =\frac{1}{2} k_{B} T
\end{aligned}
$$

The pressure due to the roton gas:

$$
p_{\rho}=-(\partial F / \partial V)_{T} \cong-F / V=n_{\rho} k_{B} T=n_{\rho} \mu\left\langle v^{2}\right\rangle
$$

\section{Free rotons}

It is worth repeating that the expression for the velocity has the curious property that $\vec{v}$ is parallel to $\vec{p}$ when $p>p_{0}$, but antiparallel when $p$ is below 
the minimum. We will refer to rotons with momentum above the minimum as $R^{+}$or ordinary, those with momentum below as $R^{-}$or extraordinary. The surface $\varepsilon(p)=\Delta$ is a sphere of radius $p_{0}$ in momentum space. For an energy greater than $\Delta$ (but below the maximum) the surface has two concentric spherical sheets, one with $p>p_{0}$, on which the rotons are ordinary, the other with $p<p_{0}$, describing extraordinary rotons. Figure 2 shows a twodimensional representation. We will often denote the two "conjugate" values of momentum with equal energy as $p$ and $\tilde{p}$.

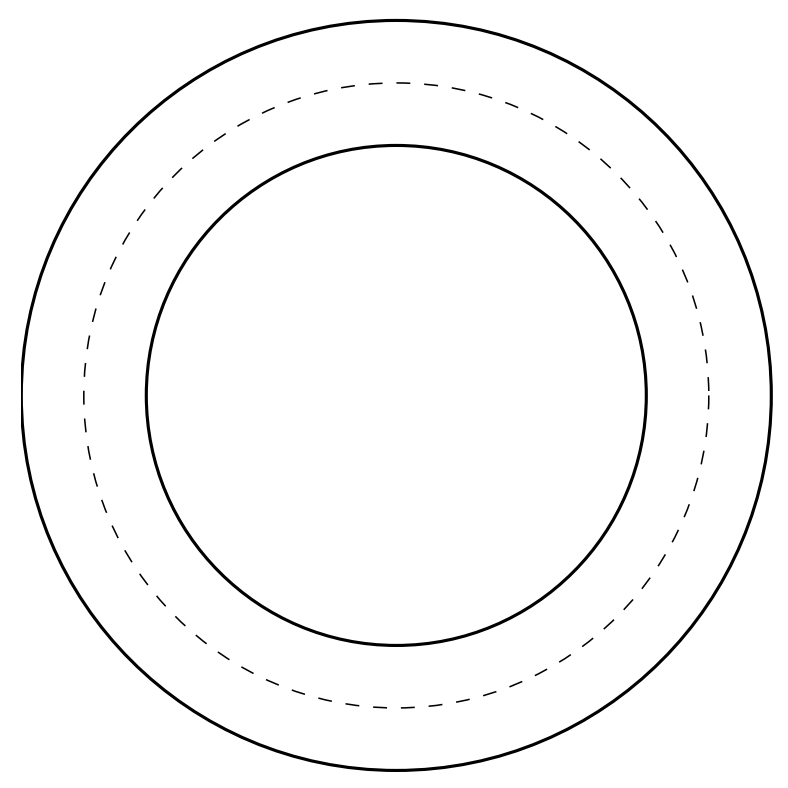

Figure 2: Surfaces of constant energy in momentum space for $\varepsilon=\Delta$ (dotted), and a higher energy (solid).

\section{Rotons in a velocity field}

A somewhat elusive argument, due to Landau, yields the result that to produce an elementary excitation of momentum $\vec{p}$ in a fluid moving at velocity $\vec{v}$ requires energy

$$
\varepsilon^{(\vec{v})}(\vec{p})=\varepsilon(\vec{p})+\vec{p} \cdot \vec{v},
$$


where $\varepsilon(\vec{p})$ is the energy of the excitation in the stationary fluid. In a slowlyvarying velocity field, we add a term to the hamiltonian

$$
\delta \varepsilon(\vec{p})=\vec{p} \cdot \vec{v}(\vec{r}) .
$$

A quasiparticle of a particular momentum magnitude has higher energy if its momentum points "downstream" than if it is directed "upstream". Thus, in thermal equilibrium, the excitations will reduce the momentum of a moving superfluid. Landau uses this argument to calculate the normal-fluid density. In section 7, we will apply it to the problem of roton motion near a quantized vortex.

We present a rather pedestrian but (we hope) straightforward derivation of this familiar result in Appendix A.1

\section{Rotons in a force field}

The roton parameters $\Delta, p_{0}$, and $\mu$ can vary with position, since they are known to depend on the density of the liquid. The "energy gap" $\Delta$, in particular, decreases from $8.62 \mathrm{~K}$ at the vapor pressure to approximately $7.2 \mathrm{~K}$ at a pressure of $25 \mathrm{~atm}$ [8]. If the roton parameters vary sufficiently slowly compared with the roton wavelength, the roton will follow a classical trajectory. Since $q_{0}=p_{0} / \hbar \approx 2 \AA^{-1}$, we have $\lambda \approx 3 \AA$; a spatial variation of $\Delta$ on a scale large compared with $3 \AA$ will appear like a classical potential energy in $\varepsilon(p) \equiv \mathcal{H}(\vec{p}, \vec{r})$. In the case in which only variation in $\Delta$ is considered, we have a Hamiltonian consisting of a "kinetic" energy $T=\left(p-p_{0}\right)^{2} / 2 \mu$, and a "potential" energy $V(\vec{r})=\Delta(\vec{r})$.

\subsection{A constant force}

An interesting illustration of the consequences of the unusual dispersion relation is provided by considering the motion of rotons in a uniform force field, i.e. when $\Delta$ is a linear function of a single coordinate, say $y$. If we write $f=-d \Delta / d y$, then Hamilton's equations take the form

$$
\begin{aligned}
\dot{p_{x}} & =-\partial \mathcal{H} / \partial x \\
& =0 \\
\dot{p_{y}} & =-\partial \mathcal{H} / \partial y
\end{aligned}
$$




$$
\begin{aligned}
& =f \\
\dot{x} & =\partial \mathcal{H} / \partial p_{x} \\
& =\left(p_{x} / p\right) \times d T / d p \\
\dot{y} & =\partial \mathcal{H} / \partial p_{y} \\
& =\left(p_{y} / p\right) \times d T / d p .
\end{aligned}
$$

The equations for $\dot{\vec{p}}$ can be integrated immediately when the force $f$ is constant. We obtain $p_{x}=p_{x i}, p_{y}=p_{y i}+f t$. On a momentum-space diagram such as that of figure 2 the motion is parallel to the $p_{y}$ axis, in a direction given by the sign of $f$. For $f<0$, we show the various possible cases in figure 3. Incident rotons with energy $\varepsilon$ and $p>p_{0}$ (ordinary rotons) exhibit three different types of trajectory, depending on the value of the angle of incidence. Extraordinary rotons (with $p<p_{0}$ ) can only become ordinary.

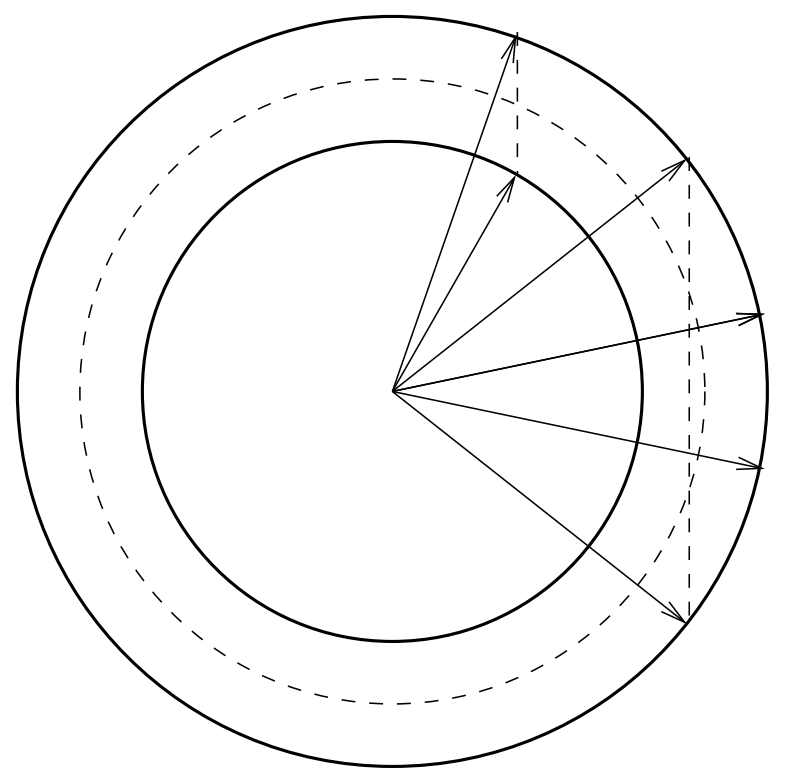

Figure 3: Momentum space diagram showing initial and final momenta corresponding to reflection of rotons by a force in the $-y$ direction.

We have $d T / d p=\left(p-p_{0}\right) / \mu$

$$
p_{x}(t)=p_{x}(0)
$$




$$
\begin{gathered}
p_{y}(t)=p_{y}(0)+f t \\
\dot{x}(t)=\left(p_{x}(0) / p(t)\right)\left(p(t)-p_{0}\right) / \mu \\
\dot{y}(t)=\left(p_{y}(t) / p(t)\right)\left(p(t)-p_{0}\right) / \mu
\end{gathered}
$$

We show in figure 1 coordinate space trajectories derived from these results. There is a constant repulsive force in the region $y>0$, no force for $y<0$. A cusp appears in the trajectory when $p$ becomes equal to $p_{0}$, at which point the velocity is zero, but the momentum vector is not. The unit of distance has been taken as the initial "kinetic" energy divided by the magnitude of the force, so that the roton stops after penetrating unit distance into the region. Three $R^{+}$rotons with momentum $1.1 p_{0}$ have been started at the point $(0,-1)$ with tangential $(x)$ momenta $1.05,0.95$, and 0.85 times $p_{0}$. The trajectory of an $R^{-}$roton is similar to the last of these, but traversed in the opposite direction. The trajectories of rotons with different values of the incident momentum would be similar to these, differing only in a scale factor in the $x$ direction.

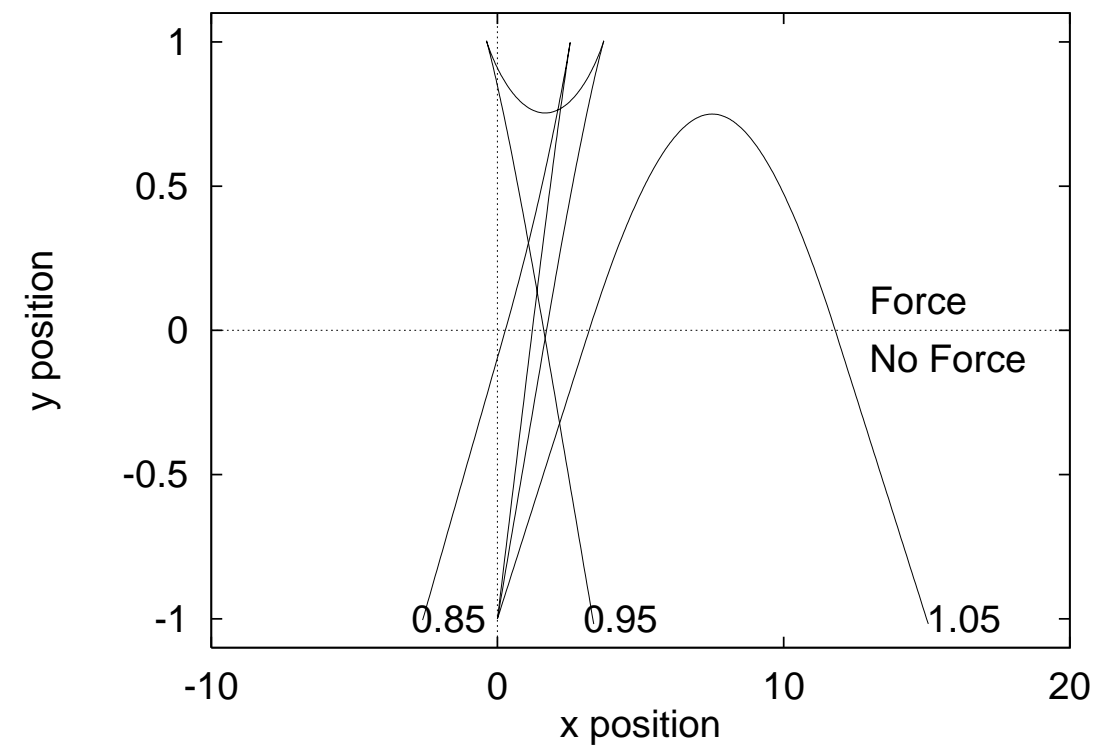

Figure 4: Coordinate space trajectories corresponding to momenta in the three regions shown in the previous figure. Notice the different scales in the two directions. 
Such cusped trajectories were apparently first noted by Goodman 10 in analyzing roton trajectories near a vortex line, a problem which we will discuss in section 17. Note, however, that these "snap back" trajectories (an apt term used in ref. [10]) have momentum transfer $\delta p$ of order

$$
\begin{aligned}
\delta p & \approx p-\tilde{p} \\
& =2\left(p-p_{0}\right)
\end{aligned}
$$

a quantity which is small compared with $2 p_{0}$. This reduces significantly the pressure exerted on a wall by a roton gas. This point has been emphasized by Maris and Cline [11].

\subsection{Pressure}

The results of the previous section imply that the pressure exerted by a roton gas must be much below what one would naïvely estimate from the flux and a momentum transfer of order $p_{0}$. As emphasized by Maris and Cline [11]. the momentum transfer must be low to agree with the exact result, given in equation 2 .

$$
p_{\rho}=-\left(\partial F_{\rho} / \partial V\right)_{T}=n_{\rho} k_{B} T .
$$

In the semiclassical case, considered here, the $R^{-}$rotons always change mode, never reflecting specularly; the $R^{+}$rotons do the same, except for nearly glancing angles. Summing the contributions to the pressure from these various processes does yield the correct result.

In quantum mechanics "Whatever is not forbidden is allowed." In particular, specular reflection is always consistent with the conservation laws, even when it does not occur classically. When we consider the branching to the other possible final state, in quantum mechanics, it can not change the result. The pressure is independent of the details of the processes at the walls. We can see explicitly that there will be cancelation between specular reflection of $R^{+}$rotons, which has large positive momentum transfer, and that of $R^{-}$ rotons, which produces a similar negative contribution to the pressure. 


\section{Rotons at a free surface}

In an early paper which considered ballistic rotons, P. W. Anderson 12 pointed out that, since the minimum roton energy $(8.6 \mathrm{~K})$ is greater than the binding energy of an atom in the liquid $\left(E_{B}=7.2 \mathrm{~K}\right)$, a roton incident on the surface might eject an atom into the vapor.

If the process is elastic, so that all the energy is transferred between the elementary excitation and the atom, and the surface is smooth, so that the component of momentum parallel to the surface is conserved, then we can define an "index of refraction" in the usual way. (Note, however, that an $R^{-}$ roton and an atom would have oppositely directed tangential velocity.)

$$
\tilde{n}=p_{A} / p=\sin \theta / \sin \phi
$$

where $p$ and $\theta$ are the momentum and angle of incidence of the elementary excitation and $p_{A}$ and $\phi$ the corresponding quantities for the helium atom. In this section, in order to connect with experiments described in section 8, we consider the entire excitation spectrum - not just the roton region.

The index $\tilde{n}$ is a single-valued function of $p$, but a multiple-valued function of $p_{A}$, since an incident atom might produce as many as three different excitations with a given energy. Written in terms of $p$ (or $q=p / \hbar$ ), we have

$$
\tilde{n}=\sqrt{\left(2 m_{4} / p^{2}\right)\left(\varepsilon(p)-E_{B}\right)}
$$

Figure 5 plots the refractive index versus momentum, and also shows the energy of the elementary excitation and a free atom of equal momentum. The index equals 1 where the two energies cross.

\section{Roton-vortex interaction}

\subsection{Quantized vortices}

The spatially-varying superfluid velocity field near a straight vortex line is:

$$
\vec{v}_{s}(\vec{r})=\vec{\kappa} \times \vec{r} / 2 \pi r^{2}=(\kappa / 2 \pi r) \hat{\varphi} .
$$

Here $\vec{r}$ is the two-dimensional (polar) radius vector, $\varphi$ the polar angle, and $\vec{\kappa}$ is along the vortex line ( $z$ direction). The definition of the circulation $\kappa$ is

$$
\kappa \equiv \oint \overrightarrow{v_{s}} \cdot \overrightarrow{d l}
$$




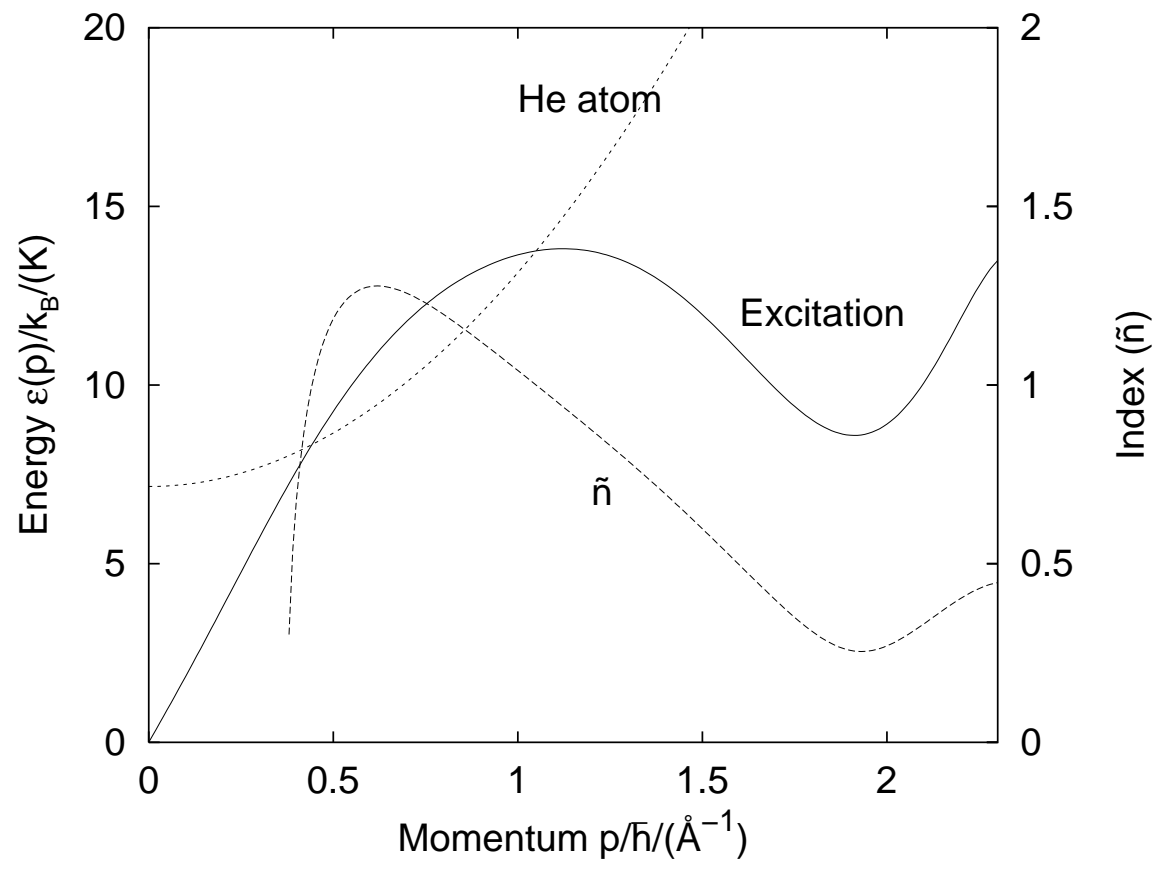

Figure 5: Energy of an elementary excitation, and an atom outside the liquid, and the "Refractive index" plotted against momentum/ $\hbar$. 
and, in the case of superfluid liquid ${ }^{4} \mathrm{He}$, is quantized in units of $h / m_{4}$. These equations are valid outside a core region, which is of atomic dimensions.

\subsection{Quasiparticle-vortex interaction}

Goodman [10] and Samuels and Donnelly [13] calculated the "mutual friction" between normal and superfluid using the roton-vortex interaction, which causes momentum transfer between the rotons (a constituent of the normal fluid) and the superfluid vortices.

If a quasiparticle is not too close to the vortex core, the velocity field will not vary very rapidly, and we can combine equations 4 and 5 to write:

$$
\delta \varepsilon(\vec{r})=\vec{p} \cdot \vec{\kappa} \times \vec{r} / 2 \pi r^{2},
$$

which can be rewritten in the form

$$
\begin{aligned}
\delta \varepsilon(\vec{r}) & =\vec{\kappa} \cdot \vec{r} \times \vec{p} / 2 \pi r^{2} \\
& =\kappa L_{z} / 2 \pi r^{2},
\end{aligned}
$$

if we take the $z$ axis along the vortex line. In this form, it is apparent that $L_{z}$, the $z$ component of angular momentum will be a constant of the motion, permitting analysis of the trajectory to be reduced to quadrature. 114

We show trajectories of $R^{+}$and $R^{-}$rotons, calculated by numerically integrating the equations of motion, in figure 6. An $R^{+}$roton with incident $x$ momentum $\left(p_{i}\right)_{x}=-1.1 p_{0}$ is started from the right, an $R^{-}$roton with $x$ momentum $\left(p_{i}\right)_{x}=0.9 p_{0}$ from the left for various values of the impact parameter. Distances are measured in units of a "critical" distance

$$
R^{c}=(\kappa / 2 \pi)\left(\mu / p_{0}\right) \frac{2 p_{i} / p_{0}}{\left[\left(p_{i} / p_{0}\right)-1\right]^{2}}=\left(1 / q_{0}\right)\left(\mu / m_{4}\right) \frac{2 p_{i} / p_{0}}{\left[\left(p_{i} / p_{0}\right)-1\right]^{2}},
$$

at which the roton-vortex interaction energy is equal to the initial roton kinetic energy, about $16 \AA$ for the rotons illustrated.

Both the $R^{+}$roton traveling to the left and the $R^{-}$roton traveling right have their momenta directed to the right. Thus, both experience a repulsive interaction above the (counter-clockwise circulating) vortex, where $\vec{p} \cdot \overrightarrow{v_{s}}$ is

positive. The mode-changing trajectories all have impact parameters on the same side of the vortex. 


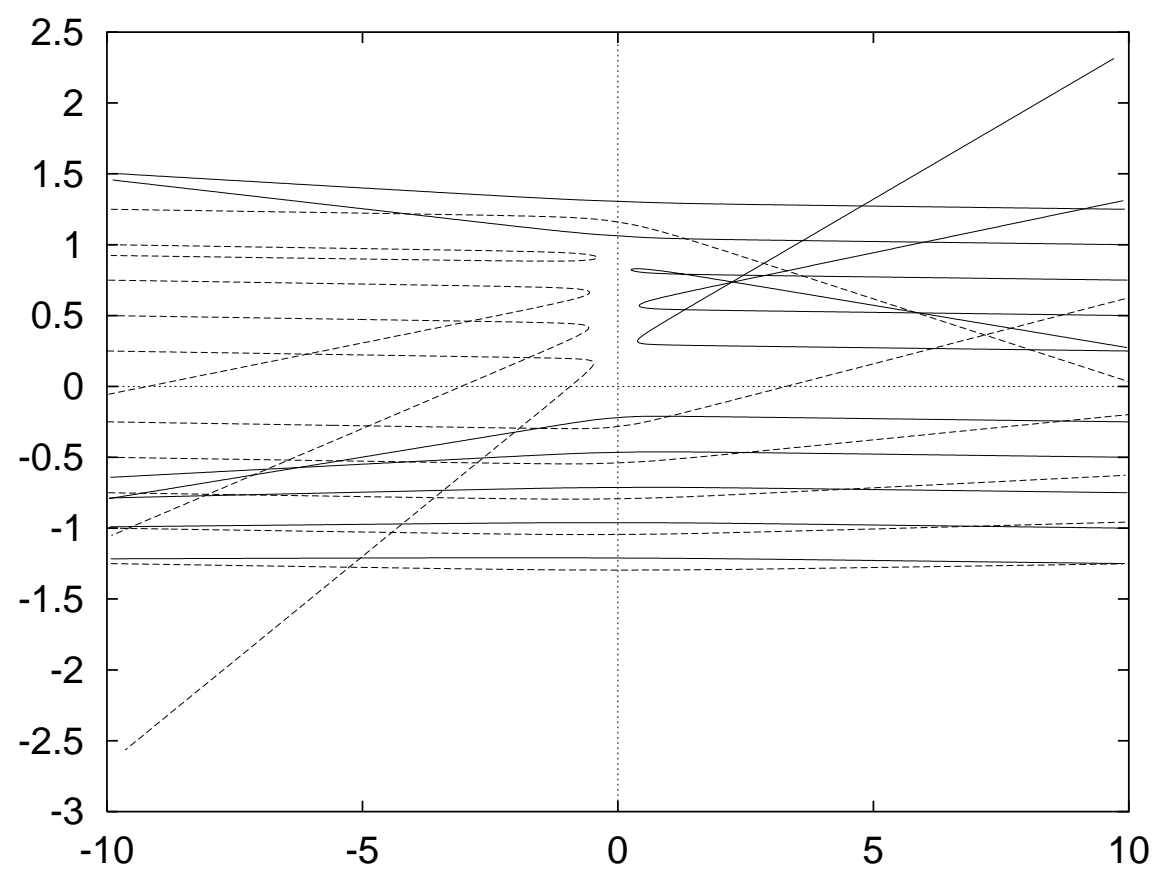

Figure 6: Calculated roton trajectories near a quantized vortex line, located at the origin. The curves are calculated for an incident $R^{+}$roton with momentum $1.1 p_{0}$ incident from the right (solid lines), and an $R^{-}$roton with momentum $0.9 p_{0}$ incident from the left (dashed lines). 


\section{The experiments}

Several experimenters performed pioneering experiments involving rotons at temperatures low enough so that the roton mean free path is long.

At Ohio State University, evidence for roton production was sought in a study of helium atom reflection from the free surface. [15]

In Paris, the inverse experiment was performed. [16] Ballistic rotons, produced at a pulsed heater 'evaporated' atoms from the free surface.

These experiments have been extended and elaborated in a series of experiments at University of Exeter. In the Exeter experiments, rotons (and phonons) are produced at a pulsed heater and detected by a superconducting bolometer. The experiments are performed at temperatures sufficiently low that the rotons may considered to be collision-free (after they leave the vicinity of the source), and to follow "ballistic" trajectories. We cite only a few of the articles here, and refer the reader to these articles for additional references. A rather comprehensive early article is. [17]

The experiments include detection and measurement of roton-roton scattering, produced by crossing two beams. 18

A puzzling aspect of these experiments is the observation that only $R^{+}$ rotons are produced at the heater, although there was evidence that $R^{-}$ rotons could be produced at surfaces.

\subsection{Direct detection of $R^{-}$rotons}

A compelling demonstration of the odd kinematic properties of the $R^{-}$roton would be evidence of oppositely-directed momentum and velocity.

Tucker and Wyatt have recently realized a source of $R^{-}$rotons, [19] using two facing heaters, and utilizing scattering of $R^{+}$rotons to produce $R^{-}$ones. They have directed these rotons at the free surface of the liquid, producing evaporation of single atoms. As noted in section 6 the $R^{-}$roton and the atom will have oppositely directed tangential velocity.

This type of confirmation of the anomalous connection between velocity and momentum of the $R^{-}$rotons [20] [21] is accomplished in the experiment pictured in Fig. 7 .

The evaporated He atom is detected in the "backward" direction, although the momentum component parallel to the surface is conserved in the transition from roton to evaporated atom. 


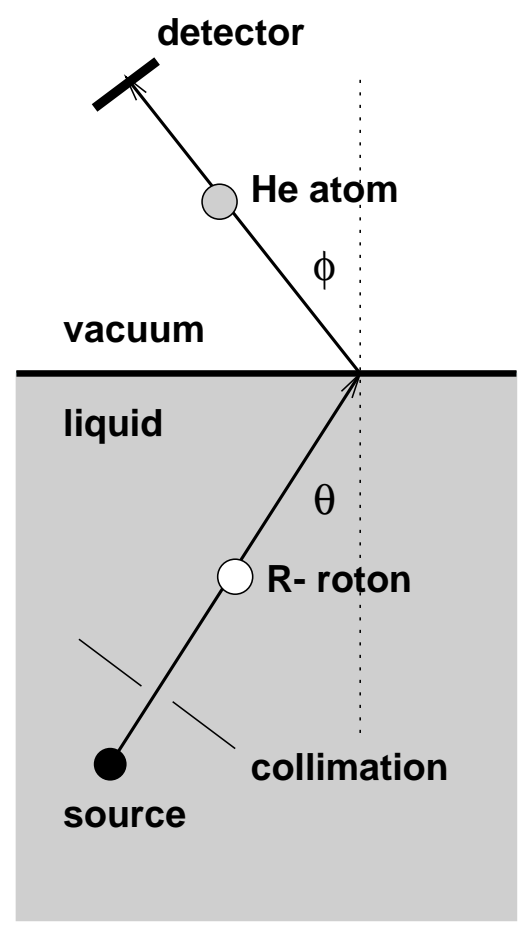

Figure 7: Evaporation of a He atom by an $R^{-}$roton. 


\section{A Appendix}

\section{A.1 Excitations in a moving fluid}

We present a rather pedestrian but (we hope) straightforward derivation of this familiar result. If we first think classically of viewing a system from a reference system in which it is moving at velocity $\vec{v}$, each particle will have $m \vec{v}$ added to its momentum, which will change the momentum and kinetic energy, but for a system which is translation-invariant (The interactions depend only on relative positions.), the potential energy will not change.

The same statements can be carried over to quantum mechanics. We can relate the wave function of our system at rest to the wave function when it has velocity $\vec{v}$ by

$$
\Psi^{(\vec{v})}=\exp \left[(m i / \hbar) \sum_{i=1}^{N} \vec{v} \cdot \overrightarrow{r_{i}}\right] \Psi
$$

where the index $i$ ranges over the $N$ atoms of the system. Since the velocity enters only the phase factor, the expectation value of the potential energy will not change.

The momentum and energy eigenvalues of the system in the two reference frames are:

$$
\begin{aligned}
& \vec{P}^{(\vec{v})}=\vec{P}+N m \vec{v} \\
& E^{(\vec{v})}=E+\vec{P} \cdot \vec{v}+N m v^{2} / 2 .
\end{aligned}
$$

Corresponding expressions for the ground state are:

$$
\begin{aligned}
& \vec{P}_{0}^{(\vec{v})}=N m \vec{v} \\
& E_{0}^{(\vec{v})}=E_{0}+N m v^{2} / 2 .
\end{aligned}
$$

For the state in which a single quasiparticle $(\vec{p}, \varepsilon)$ is present:

$$
\begin{aligned}
\vec{P}_{p}^{(\vec{v})} & =\vec{p}+N m \vec{v} \\
E_{\vec{p}}^{(\vec{v})} & =E_{0}+\varepsilon(\vec{p})+\vec{p} \cdot \vec{v}+N m v^{2} / 2 .
\end{aligned}
$$

Thus the change in momentum and energy associated with the single quasiparticle is

$$
\vec{p}^{(\vec{v})}=\vec{P}_{\vec{p}}^{(\vec{v})}-\vec{P}_{0}^{(\vec{v})}
$$




$$
\begin{aligned}
& =\vec{p} \\
\varepsilon^{(\vec{v})}(\vec{p}) & =E_{\vec{p}}^{(\vec{v})}-E_{0}^{(\vec{v})} \\
& =\varepsilon(\vec{p})+\vec{p} \cdot \vec{v}
\end{aligned}
$$

which is our equation 3 of section 4 


\section{Figure captions}

Figure 1 Dispersion curve for elementary excitations in liquid helium, calculated from the polynomial fit in reference [9]. The roton region is near the minimum.

Figure 2 Surfaces of constant energy in momentum space for $\varepsilon=\Delta$ (dotted), and a higher energy (solid).

Figure 3 Momentum space diagram showing initial and final momenta corresponding to reflection of rotons by a force in the $-y$ direction.

Figure 4 Coordinate space trajectories corresponding to momenta in the three regions shown in the previous figure. Notice the different scales in the two directions.

Figure 5 Energy of an elementary excitation, and an atom outside the liquid, and the "Refractive index" plotted against momentum/ $\hbar$.

Figure 6 Calculated roton trajectories near a quantized vortex line, located at the origin. The curves are calculated for an incident $R^{+}$roton with momentum $1.1 p_{0}$ incident from the right (solid lines), and an $R^{-}$roton with momentum $0.9 p_{0}$ incident from the left (dashed lines).

Figure 7 Evaporation of a He atom by an $R^{-}$roton. 


\section{References}

[1] L. D. Landau. The theory of superfluidity of Helium II. J. Phys. (USSR), 5:71, 1941.

[2] L. D. Landau. On the theory of supefluidity of Helium II. J. Phys. (USSR), 11:91, 1947.

[3] D. ter Haar, editor. Men of physics: L. D. Landau. Pergamon, Oxford, New York, 1965.

[4] I. M. Khalatnikov. An introduction to the theory of superfluidity. Addison-Wesley, Redwood City, CA, 1988.

[5] Russell Donnelly. Rotons: a low-temperature puzzle. Phys. World, 10:25-30, 1997.

[6] T. Ellis and P. V. E. Mcclintock. The breakdown of superfluidity in liquid ${ }^{4} \mathrm{He} \mathrm{V}$. Measurement of the Landau critical velocity for roton creation. Phil. Trans. Roy. Soc. Lond. Ser. A, 315(1532):259-300, 1985.

[7] R. P. Feynman. Prog. Low. Temp. Phys., volume I, chapter II: Application of Quantum Mechanics to Liquid Helium, pages 17-53. NorthHolland, Amsterdam, 1955.

[8] R. J. Donnelly, J. A. Donnelly, and R. N. Hills. Specific heat and dispersion curve for Helium II. J. Low Temp. Phys., 44:471-489, 1981.

[9] J. S. Brooks and R. J. Donnelly. The calculated properties of Helium II. J. Phys. Chem. Ref. Data, 6:51, 1977.

[10] S. E. Goodman. Classical model of the roton-quantized vortex interaction with an application to rotating liquid He II. Phys. Fluids, 14:1293, 1971.

[11] H. J. Maris and Richard W. Cline. Roton second sound and roton scattering. Phys. Rev. B, 23:3308, 1981.

[12] P. W. Anderson. Interpretation of the Johnston-King experiment on the evaporation of liquid ${ }^{4}$ He. Phys. Lett., 29A:563, 1969. 
[13] David C. Samuels and Russell J. Donnelly. Dynamics of the interactions of rotons with quantized vortices in Helium II. Phys. Rev. Lett., 65:187, 1990 .

[14] T. M. Sanders, Jr. Comment on "Dynamics of the Interactions of Rotons with Quantized Vortices in Helium II". Phys. Rev. Lett., 66:241, 1991.

[15] D. O. Edwards, P. Fatouros, G. G. Ihas, P. Mrozinski, S. Y. Shen, F. M. Gasparini, and C. P. Tam. Specular reflection of ${ }^{4} \mathrm{He}$ atoms from the surface of liquid ${ }^{4}$ He. Phys. Rev. Lett., 34:1153-1156, 1975.

[16] S. Balibar, J. Buechner, B. Castaing, C. Laroche, and A. Libchaber. Observation of a maximum roton velocity in superfluid ${ }^{4}$ He. Phys. Lett., 60A:135-136, 1977.

[17] M. Brown and A. F. G. Wyatt. Boundary conditions for quantum evaporation. J. Phys. Condens. Matter, 2:5025-46, 1990.

[18] A. C. Forbes and A. F. G. Wyatt. Roton-roton scattering in liquid ${ }^{4}$ He: A direct observation. Phys. Rev. Lett., 64:1393, 1990.

[19] M. A. H. Tucker and A. F. G. Wyatt. A fast pulsed source of ballistic $R^{-}$rotons in superfluid ${ }^{4}$ He. J. Low Temp. Phys., 110:455-460, 1998.

[20] M. A. H. Tucker and A. F. G. Wyatt. Direct evidence for $R^{-}$rotons having antiparallel momentum and velocity. Science, 283:1150-1152, 1999 .

[21] M. A. H. Tucker and A. F. G. Wyatt. Evaporation probability of $R^{-}$ rotons relative to $R^{+}$rotons in superfluid ${ }^{4}$ He. J. Low Temp. Phys., 113:615-620, 1998. 